ARTICLE

DOI: $10.1038 /$ s41467-018-03978-3

\title{
Crystalline polymer nanofibers with ultra-high strength and thermal conductivity
}

\author{
Ramesh Shrestha', Pengfei Li ${ }^{1}$, Bikramjit Chatterjee ${ }^{2}$, Teng Zheng ${ }^{3}$, Xufei Wu ${ }^{3}$, Zeyu Liu ${ }^{3}$, Tengfei Luo ${ }^{3}$,
} Sukwon Choi ${ }^{2}$, Kedar Hippalgaonkar ${ }^{4}$, Maarten P. de Boer ${ }^{1} \&$ Sheng Shen ${ }^{1}$

Polymers are widely used in daily life, but exhibit low strength and low thermal conductivity as compared to most structural materials. In this work, we develop crystalline polymer nanofibers that exhibit a superb combination of ultra-high strength (11 $\mathrm{GPa}$ ) and thermal conductivity, exceeding any existing soft materials. Specifically, we demonstrate unique low-dimensionality phonon physics for thermal transport in the nanofibers by measuring their thermal conductivity in a broad temperature range from 20 to $320 \mathrm{~K}$, where the thermal conductivity increases with increasing temperature following an unusual $\sim T^{1}$ trend below 100 $\mathrm{K}$ and eventually peaks around $130-150 \mathrm{~K}$ reaching a metal-like value of $90 \mathrm{~W} \mathrm{~m}^{-1} \mathrm{~K}^{-1}$, and then decays as $1 / T$. The polymer nanofibers are purely electrically insulating and bio-compatible. Combined with their remarkable lightweight-thermal-mechanical concurrent functionality, unique applications in electronics and biology emerge.

\footnotetext{
${ }^{1}$ Department of Mechanical Engineering, Carnegie Mellon University (CMU), Pittsburgh, PA 15213, USA. ${ }^{2}$ Department of Mechanical and Nuclear Engineering, Pennsylvania State University, State College, PA 16802, USA. ${ }^{3}$ Department of Aerospace and Mechanical Engineering, University of Notre Dame, Notre Dame, IN 46556, USA. ${ }^{4}$ Institute of Materials Research and Engineering, Agency for Science Technology and Research, \#08-03, 2 Fusionopolis Way, Innovis 138634, Singapore. Correspondence and requests for materials should be addressed to M.P.d.B. (email: mpdebo@andrew.cmu.edu) or to S.S. (email: sshen1@cmu.edu)
} 
$\mathrm{n}$ pursuit of lightweight multi-functional materials for aerospace, automotive, and bio-medical applications, polymers have emerged as a promising platform because they are lowdensity, easily processable, cheap, and electrically insulating. Yet, bulk polymers are partially amorphous with randomly coiled molecular chains and have defects such as voids, chain ends and entanglements. These defects act as stress concentrators and phonon scattering sites, leading to their low tensile strength $\left(\sigma_{\mathrm{ts}}, 20-100 \mathrm{MPa}\right)$, low stiffness $(E, 1-5 \mathrm{GPa})$ and low thermal conductivity $\left(k, 0.1-0.4 \mathrm{~W} \mathrm{~m}^{-1} \mathrm{~K}^{-1}\right)^{1}$. On the other hand, just as a spider synthesizes silk from protein polymer to form a fiber with $\sigma_{\mathrm{ts}}$ similar to high tensile strength steel ${ }^{2}$, polymers can be spun and drawn to form stiff high-strength materials with exceptionally high $k^{3-5}$. In particular, at the nanoscale, polymer chains can become highly oriented, and the defects that lower $\sigma_{\text {ts }}$ and $k$ can be significantly reduced ${ }^{6-8}$. This leads to simultaneous enhancement in $\sigma_{\mathrm{ts}}$ and $k$, which is different from turbostratic carbon fibers where randomly folded and interlinked graphitic domains enhance $\sigma_{\mathrm{ts}}$ but suppress $k^{9-11}$.

Fiber processing techniques such as gel spinning ${ }^{3-5}$, tip drawing $^{6,7}$ and electrospinning ${ }^{12}$ have been developed to enhance crystallinity and chain alignment and thus engineer the thermal and mechanical properties of polymers. Commercial oriented PE gel spun microfibers, with diameters ranging from $10-20 \mu \mathrm{m}$, demonstrated enhanced $k$ up to $20 \mathrm{~W} \mathrm{~m}^{-1} \mathrm{~K}^{-1}$ at room temperature $(\mathrm{RT})^{4}$, as well as a $\sigma_{\mathrm{ts}}$ of $3.9 \mathrm{GPa}$ (Dyneema ${ }^{\circledR} /$ Royal DSM). Electrospinning can produce nanoscale PE fibers with diameters as small as $50 \mathrm{~nm}$, but only moderate crystallinity and alignment were achieved with $k$ up to $9 \mathrm{~W} \mathrm{~m}^{-1} \mathrm{~K}^{-1}$ at $300 \mathrm{~K}^{12}$. By optimizing the gel electrospinning process at an elevated temperature, $\sigma_{\text {ts }}$ up to $6.3 \mathrm{GPa}$ on a fiber of $490 \mathrm{~nm}$ diameter was achieved $^{13}$. Although tip-drawn PE nanofibers have been reported to have a RT $k$ as high as $104 \mathrm{~W} \mathrm{~m}^{-1} \mathrm{~K}^{-16}$, temperaturedependent thermal transport in such ultra-drawn nanofibers, which is critical for not only elucidating the phonon physics in the nanofibers but also developing their applications in a wide temperature range, remains unknown. Recently, a near theoretical $E$ of $312 \mathrm{GPa}$ has been reported in tip-drawn PE nanofibers ${ }^{7}$. However, $\sigma_{\mathrm{ts}}$ of such polymers, which is the limiting factor in many applications, needs to be explored.

Here, we report a fabrication method that can consistently produce polyethylene (PE) nanofibers with diameters ranging from 10 to $100 \mathrm{~nm}$. We measure $k$ over a broad temperature range from 20 to $320 \mathrm{~K}$. We find that $k$ increases with temperature, following an unusual $T^{1}$ trend below $100 \mathrm{~K}$ eventually peaking around $130-150 \mathrm{~K}$, and reaching a value as high as $90 \mathrm{~W}$ $\mathrm{m}^{-1} \mathrm{~K}^{-1}$. Thereafter, $k$ decays as $1 / T$. Measurements show ultrahigh $\sigma_{\mathrm{ts}}, 11.4 \pm 1.1 \mathrm{GPa}$, for our crystalline PE nanofibers. To the best of our knowledge, this is the highest $\sigma_{\mathrm{ts}}$ measured for any polymer based fibers, including PE fibers (Dyneema ${ }^{\circledR} /$ Royal DSM and Spectra $^{\circledR} /$ Honeywell), aramid fibers (Kevlar ${ }^{\circledR} /$ DuPont), carbon fibers and composite fibers ${ }^{13-16}$.

\section{Results}

Two-step fabrication of crystalline PE nanofibers by local heating. The fabrication of PE nanofibers, PENFs, was conducted in two steps where a microfiber, PEMF, was tip drawn from a PE/ decalin gel and then further stretched to the nanometer scale by localized heating in air (see Methods and Supplementary Note 2). As shown in Fig. 1, a small segment of a pre-stressed PEMF was placed near a simple micro heater, and locally heated near the melting point. The pre-existing tensile stress quickly stretched the fiber (see Supplementary Movie 1) to a diameter in the range of $10-100 \mathrm{~nm}$, which could be controlled by tuning the pre-existing stress or the heater temperature. Per the schematic in Fig. 1a, amorphous regions are stretched and recrystallized, leaving a nearly defect-free aligned crystalline PENF.

The local heating method developed in this work has a number of advantages over the regular heating method, where the whole fiber is heated and stretched over an oven or hot plate until reaching a nanoscale diameter ${ }^{6,7}$. The $k$ of $\mathrm{PE}$ is directly correlated with the crystallinity and molecular alignment along the fiber axis of the sample. The average extension of molecules increases with the strain rate ${ }^{17}$. With the drawing by local heating, a strain rate up to $1400 \mathrm{~s}^{-1}$ can be achieved (see Supplementary Note 2) compared to $\sim 1000 \mathrm{~s}^{-1}$ in gel electrospinning ${ }^{13}$ and $\sim 1 \mathrm{~s}^{-1}$ in gel spinning ${ }^{5}$. Another important aspect is the relaxation of molecules, which competes with stretching. In local heating, the fiber is rapidly quenched to minimize the relaxation. The resulting high degree of alignment, coupled with high crystallinity, leads to the improved $\sigma_{\text {ts }}$ and $k$. The fiber drawing by local heating yields a segment of nanofiber $(\sim 100 \mu \mathrm{m}$ long) that spans between microfibers. Using the microfiber segments for tracking, the nanofiber can be precisely manipulated onto test platforms. In addition, the fabrication yield is much a

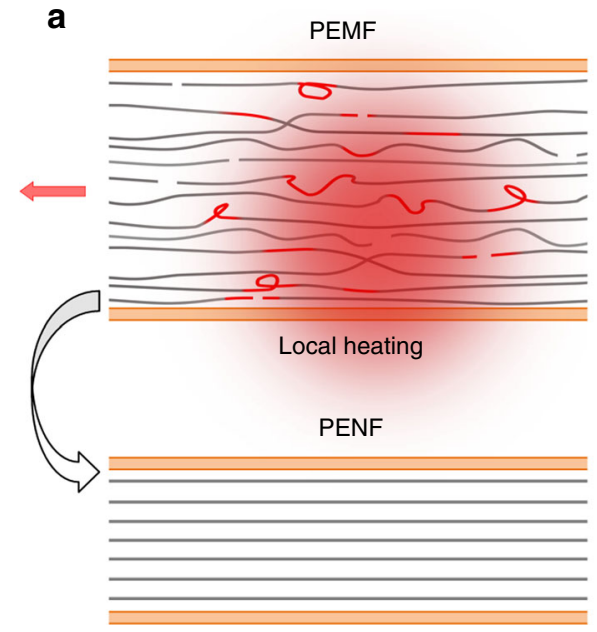

b

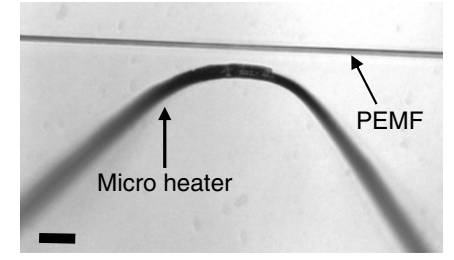

C

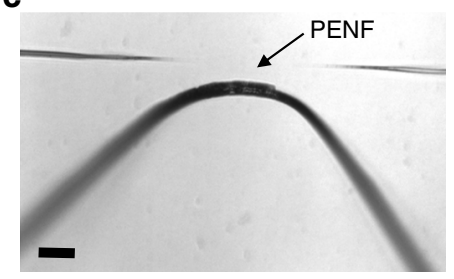

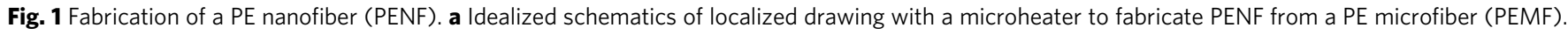
Arrows indicate drawing directions whereas red lines highlight the defects in PEMF. b-c Optical micrograph before and after localized drawing (in c, nanofiber not resolved optically). Scale bars, $20 \mu \mathrm{m}$ 
a

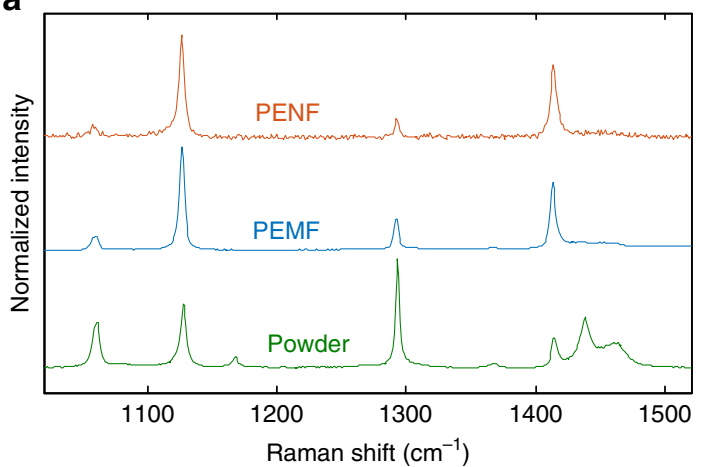

b

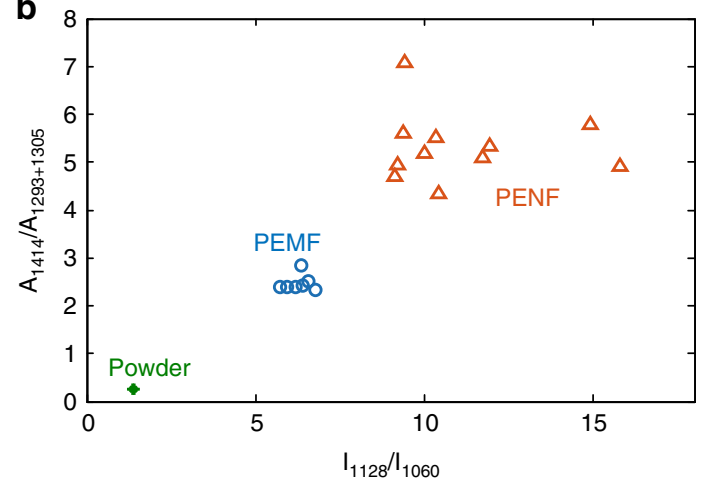

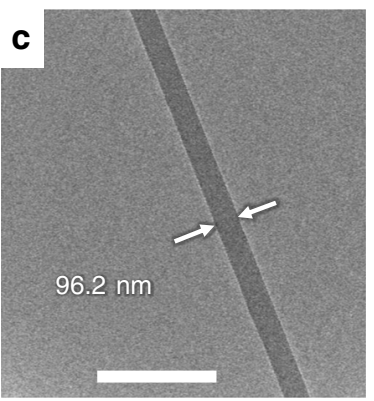
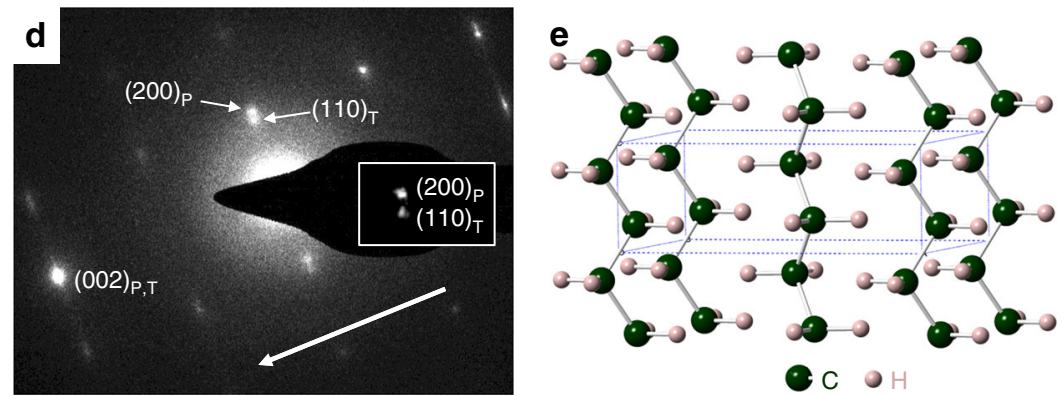

Fig. 2 Morphological and structural characterizations. a Micro-Raman spectra of PE powder, PEMF and PENF where the data is normalized to the highest peak of each spectrum. $\mathbf{b}$ Ratio of integral areas of Raman band $1414 \mathrm{~cm}^{-1}$ to Raman bands $1293 \mathrm{~cm}^{-1}$ and $1305 \mathrm{~cm}^{-1} \mathrm{vs}$. intensity ratio of Raman bands $1128 \mathrm{~cm}^{-1}$ to $1060 \mathrm{~cm}^{-1}$. c TEM micrograph of a PENF. d SAED pattern of the PENF in c Inset shows the distinct diffraction spots of (200) plane of parent crystal (subscript P) and (110) plane of twinned crystal (subscript T). Arrow indicates the drawing direction. e Orthorhombic crystal structure of PE. Scale bar, $500 \mathrm{~nm}$

higher with local drawing. In regular drawing, hot air flow rising from the hot plate/oven makes the fiber stretching susceptible to local hot spots. A fiber with various hot spots is more likely to have different local stress and strain, increasing the likelihood of premature breakage.

Crystal structure of PE nanofibers. To characterize the PENF crystal structure, we employed micro-Raman and low-dose transmission electron microscopy at cryogenic temperatures (cryo-TEM). The micro-Raman was used to understand the orientation and crystallinity of PE during different stages of drawing. A qualitative measure of molecular orientation can be obtained from the peak intensity ratio of $1128 \mathrm{~cm}^{-1}$ and $1060 \mathrm{~cm}^{-1}$ Raman bands ${ }^{18}$. Similarly, the crystallinity can be measured by the ratio of the integral areas under Raman bands of $1414 \mathrm{~cm}^{-1}$ to $1293 \mathrm{~cm}^{-1}$ and $1305 \mathrm{~cm}^{-1}$, where Raman band of $1414 \mathrm{~cm}^{-1}$ and Raman bands of $1293 \mathrm{~cm}^{-1}$ and $1305 \mathrm{~cm}^{-1}$ correspond to the orthorhombic crystal and an internal standard independent of chain conformation, respectively ${ }^{18}$. Figure 2 a shows typical Raman spectra of PE powder, PEMF and PENF. The spectra show that the area under the $1414 \mathrm{~cm}^{-1}$ band increases from powder to PEMF and then PENF indicating that the amorphous phase is significantly reduced and crystallization is promoted (see Supplementary Note 2 for crystallinity of PE powder). In addition to crystallinity, molecular orientation also increases from powder to PEMF and then PENF (Fig. 2b).

The structural and morphological properties of PENF were further characterized using low-dose cryo-TEM. Figure $2 \mathrm{c}$ shows a TEM micrograph of a typical PENF sample. Selected area electron diffraction (SAED) patterns (Fig. 2d) with an orthorhombic crystal structure (Fig. 2e) of crystalline PE with lattice constants $a=7.301 \AA, b=4.893 \AA$ and $c=2.550 \AA$ were indexed. These constants agree well with literature values from experiments and molecular simulations ${ }^{19-21}$. The arrow indicates the drawing direction, which is along the $c$-axis, and shows that (001) remains along the fiber axis. During the highly localized heating and drawing, the PEMF is stretched at a high strain rate while undergoing plastic deformation, which can occur via slip, twinning or martensitic phase transformation ${ }^{22,23}$. Among the principal $\{110\}$ and $\{310\}$ twin planes that can be activated in PE, $\{310\}$ twinning may occur at high strain under which the morphology changes from lamellar to fibrillar ${ }^{24}$. The SAED pattern (Fig. 2d) indicates that in oriented PENF $\{310\}$ twinning occurs. Because the twinning involves rotations about the $c$-axis $\left(54^{\circ}\right)$ and the properties in this work are measured along that axis, the effects are expected to be negligible. The presence of a monoclinic phase was also observed in SAED pattern but its volume fraction is small (see Supplementary Fig. 8).

Thermal transport in crystalline PENF. We measured the temperature-dependent $k(T)$ of PENF in high vacuum $\left(2 \times 10^{-7}\right.$ Torr) using a suspended platinum resistance thermometer (PRT) microdevice (see Fig. 3a), which consists of heating and sensing measurement islands ${ }^{25-27}$. To achieve a low thermal contact resistance, a platinum or graphite coating deposited by electron or focused ion beam (FIB) has been extensively used in literature ${ }^{28,29}$. However, these methods cannot be applied in our case due to the sensitivity of PENFs to high energy electron/ion beams, both of which severely damage the crystallinity and molecular orientation of the samples ${ }^{30}$ (see Fig. $3 \mathrm{~d}$ and Supplementary Fig. 13). To enhance the thermal contact between PENF and the device, we exploited a technique we call capillary-assisted adhesion of PENF by first placing an isopropanol (IPA) drop on top of the thermal device. Surface tension from the evaporating liquid then pulls the PENF into intimate contact with the pads.

The equivalent PENF thermal circuit can be expressed as a series of thermal resistances, namely intrinsic thermal resistance 
a

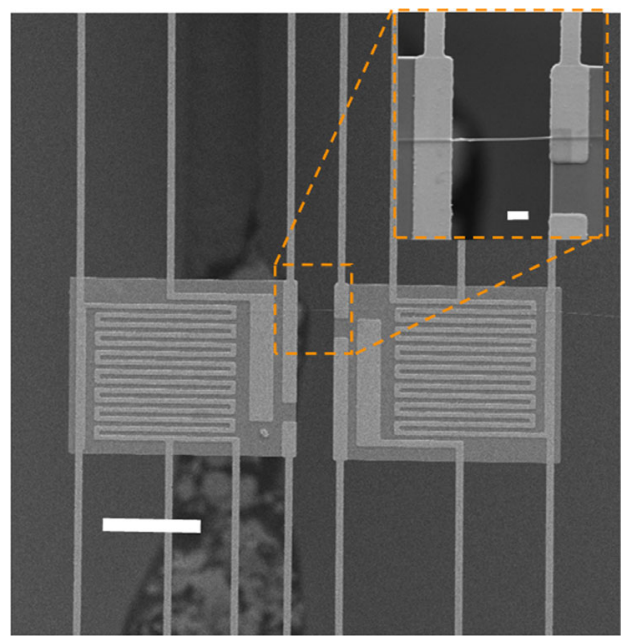

b

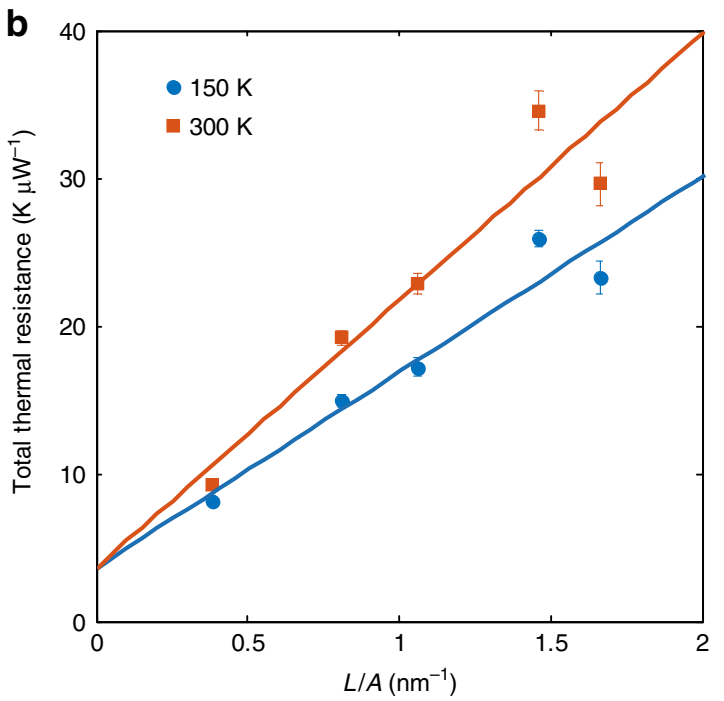

C

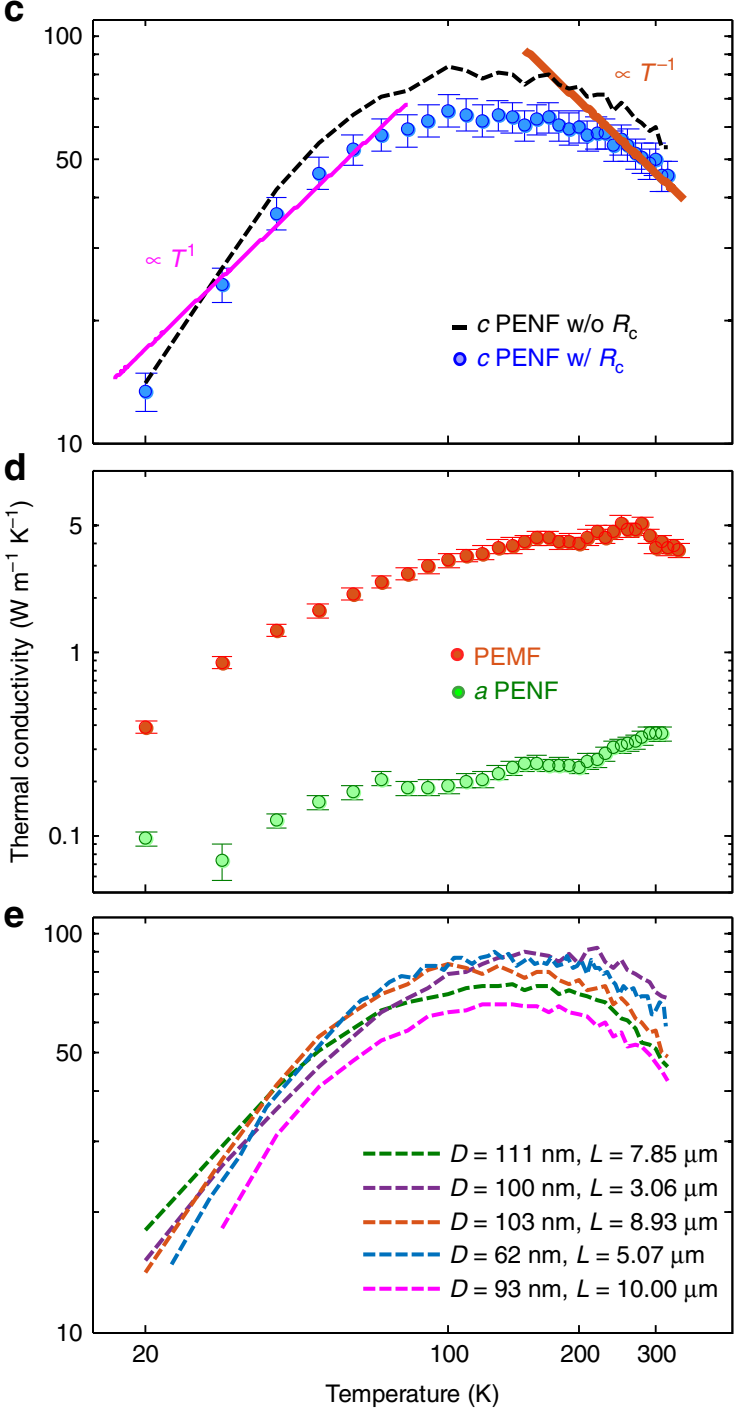

Fig. 3 Thermal characterization of PENF. a A PRT based micro-device with the heating and the sensing islands. Inset shows a PENF bridging the islands. $\mathbf{b}$ Total measured thermal resistance vs. $L / A$ of crystalline (c) PENFs at $150 \mathrm{~K}$ and $300 \mathrm{~K}$ where the $y$-intercept represents $2 R_{\mathrm{c}}$. The error bars show the mean and standard deviation values of 250 measurements. $\mathbf{c} k(T)$ of a PENF. Blue circles are measured $k$ of a c-PENF, black dotted lines are after adjusting for $2 R_{c}$. Orange line is $1 / T$ trend of $c$-PENF at high temperatures and pink line is $T^{1}$ trend at low temperatures. The error bars are obtained using error propagation in $2 R_{\mathrm{c}}$ and measured diameter (see Supplementary Note 4 for detailed uncertainty analysis). $\mathbf{d} k(T)$ of PEMF and amorphous (a) PENF. Red circles are PEMF before local drawing, and green circles are c-PENF amorphized due to exposure to FIB. e $k(T)$ of all reported samples after adjusted for $2 R_{\mathrm{c}}$. Scale bars, 20 $\mu \mathrm{m}$ and $2 \mu \mathrm{m}$ (inset)

of the nanofiber $R_{\mathrm{s}}$, thermal contact resistance between the nanofiber and the heating island $R_{\mathrm{c}, \mathrm{hi}}$ and the sensing island $R_{\mathrm{c}, \mathrm{si}}$. The heating and sensing islands have the same geometrical and material properties and undergo the same contact mechanism, so we can assume that $R_{\mathrm{c}, \mathrm{hi}} \approx R_{\mathrm{c}, \mathrm{si}} \approx R_{\mathrm{c}}$. The measured $R_{\text {tot }}$ is the total thermal resistance of this circuit. Therefore,

$$
R_{\mathrm{tot}}=R_{\mathrm{s}}+2 R_{\mathrm{c}}=\frac{L}{k A}+2 R_{\mathrm{c}}
$$

where $L$ is the gap length between the heating and the sensing islands, $A$ is the cross-sectional area of the fiber and $2 R_{\mathrm{c}}$ is the total thermal contact resistance. Assuming similar $k$ for the samples with similar diameter, we estimate $2 R_{\mathrm{c}}$ by extrapolating the linear fit between $R_{\text {tot }}$ and $L / A$ to y-intercept. The linear fits (Fig. $3 \mathrm{~b}$ ) at $150 \mathrm{~K}$ and $300 \mathrm{~K}$ show that $2 R_{\mathrm{c}}$ is $3.6 \times 10^{6} \mathrm{~K} \mathrm{~W}^{-1}$ and is approximately temperature-independent above $100 \mathrm{~K}^{31}$.
This value is consistent with $\left(2 R_{\mathrm{c}} \approx 4 \times 10^{6} \mathrm{~K} \mathrm{~W}^{-1}\right)$ predicted using a line-contact model (see Supplementary Fig. 14). Figure $3 c$ shows the $k(T)$ of a typical nanofiber, which increases as $\sim T^{1}$ and reaches a maximum of $\sim 90 \mathrm{~W} \mathrm{~m}^{-1} \mathrm{~K}^{-1}$ at $130 \mathrm{~K}-160 \mathrm{~K}$. It then decreases as $1 / T$ on further increasing the temperature. RT values were $50-70 \mathrm{~W} \mathrm{~m}^{-1} \mathrm{~K}^{-1}$ (see Fig. 3e), which is on the same order as those of tip drawn nanofibers ${ }^{6}$. This is a significant enhancement $(10-14 \times)$ using local drawing as the PEMF RT $k$ is $5 \mathrm{~W} \mathrm{~m}^{-1} \mathrm{~K}^{-1}$ (see Fig. 3d). However, PENFs with similar diameter show scatter in RT $k$ values which may be because of the variation in crystallinity and orientation among the nanofibers as shown in Fig. 2b. Recent studies on ultra-drawn PEMF and films have also obtained $k$ around $50-60 \mathrm{~W} \mathrm{~m}^{-1} \mathrm{~K}^{-1}$ at $\mathrm{RT}^{32-34}$. After careful characterization on the cross section of the PENFs, we observed that samples with diameters below $150 \mathrm{~nm}$ generally have a non-circular cross-section (See Supplementary Fig. 21, where we provide extensive documentation detailing how this was 
a

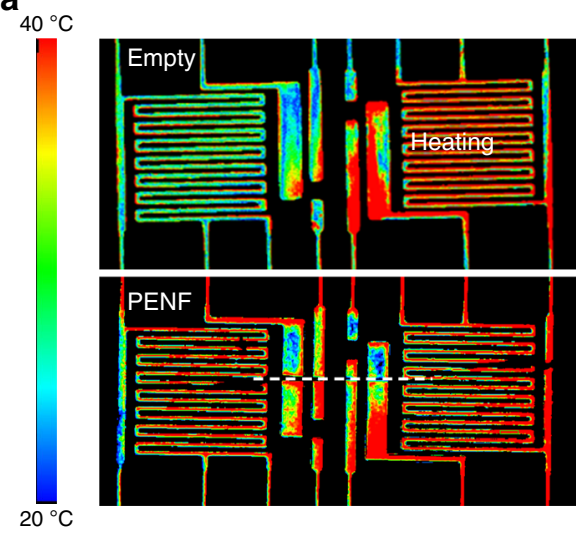

b

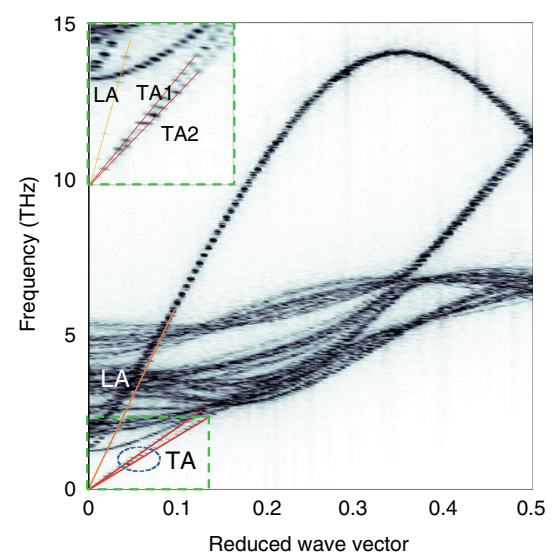

Fig. 4 Thermal transport in PENF. a Thermoreflectance imaging of heating island (right) and sensing island (left) without a nanofiber and a PENF respectively, in laboratory air. Dotted white line is the PENF position. b Phonon dispersion relation of the acoustic branches along the chain backbone calculated from the trajectory of MD simulations using two-dimensional Fourier transform. The contour is the raw data from calculation and colored lines are linear fits. The inset shows the acoustic branches close to the Brillouin zone center

characterized), in which the measured height is about 1.5 times smaller than the measured width. We assumed the fibers to have a cylindrical shape and used the measured width for calculating $k$ of PENFs from the measured thermal conductance. Hence, the $k$ reported in Fig. $3 c$ and e should be taken as a lower bound.

To directly demonstrate the heat transfer along the PENF we used high-resolution thermoreflectance imaging (Microsanj NT210B) to precisely map the temperature distribution of the micro thermal device in Fig. 4a, in which the island on the right-hand side was electrically heated. We compared the following two cases for the same batch of devices at the same heating power in laboratory air. In the absence of a nanofiber bridging the two islands, the left island was heated negligibly due to the low thermal conductivity of the air gap (Fig. 4a). In the case of our fabricated PENF with a diameter of $\sim 500 \mathrm{~nm}$ (Fig. 4a), the left island of the micro device was dramatically thermalized because of the extremely high thermal conductivity $\left(\sim 70 \mathrm{~W} \mathrm{~m}^{-1} \mathrm{~K}^{-1}\right)$. This experiment demonstrated that the PENF can potentially be used as efficient heat spreaders for microelectronics. Importantly, compared with existing nanoscale heat spreaders like carbon nanotubes and graphene, the PENF have a practical advantage because they are purely electrically insulating.

While it is well understood that the $1 / T$ relation of $k$ at high temperatures is due to anharmonic phonon scattering in crystals, the $T^{1}$ relation at low temperatures is uncommon in crystalline materials ${ }^{28,35}$. To understand this unusual $k(T)$ trend of PENFs, we performed atomistic simulation to analyze the phonon properties in a perfect PE crystal with extended chains (see Methods). We first calculated the phonon dispersion relation along the chain backbone from molecular dynamics simulations (Fig. 4b). Similar to common 3D crystals, there are three acoustic phonon branches, with each being linear close to the Brillouin zone center. If we consider the relaxation time approximation for the phonon Boltzmann Transport Equation, we can use the following equation to express the thermal conductivity:

$$
k=\sum_{\mathbf{q}, \alpha} c_{\mathbf{q}, \alpha} v_{\mathbf{q}, \alpha} l_{\mathbf{q}, \alpha}
$$

where $c_{\mathbf{q}, \alpha}, v_{\mathbf{q}, \alpha}$, and $l_{\mathbf{q}, \alpha}$ are respectively specific heat, group velocity and mean free path of phonon with wavevector $\mathbf{q}$ and polarization $\alpha$ along the chain backbone direction. This is a scalar $k$ along the chain backbone direction which can also be deduced from the more general tensor form ${ }^{36}$. At low temperatures, where the anharmonic phonon scattering is weak, the mean free path is usually limited by extrinsic scattering mechanisms, such as defect and boundary scattering. In our case, these can be from polymer chain ends or other defects along the chain backbone, such as segment rotation, which are much stronger scattering centers than point defects ${ }^{21,37}$. Under such a condition, we assume that the mean free path is the same for all phonons, which is also a common assumption made for $3 \mathrm{D}$ crystals at low temperatures ${ }^{38}$. As a result, $k(T)$ is purely from the heat capacity. For the linear phonon branches in a 1D system, $k$ should scale as $T^{138}$. Thermal transport in aligned PE chain bundles is highly anisotropic and is dominated by the chain backbone since the inter-chain vdW interactions are much weaker than the covalent bonding along the backbone. However, phonons with wavevectors not strictly aligned along the chain backbone direction can still contribute to $k$ though in a much smaller proportion. This $3 \mathrm{D}$ effect of phonon dispersion can result in $k$ scaling slightly greater than $T^{1}$ at the low temperature limit, which can be seen in Fig. 3c. At the high temperature end, the experimental data also matches well with results from early MD simulations ${ }^{37}$ where non-equilibrium MD was used to calculate the $k$ of crystalline PE. The high temperature data can be reasonably fit by a $1 / T$ relation.

Ultra-high $\sigma_{\text {ts }}$ of crystalline PENF. The $\sigma_{\text {ts }}$ of individual nanofibers was tested in tension using a microelectromechanical systems (MEMS) based device with an on-chip actuator that is a micro stepper motor with $60 \mathrm{~nm}$ step size and large in-plane force (up to $1 \mathrm{mN})^{39,40}$. The actuator displacement range is $>100 \mu \mathrm{m}$ and can apply large strain on a $30 \mu \mathrm{m}$ nominal gauge length nanofiber. The metrology based on optical microscopy has a displacement resolution of $4 \mathrm{~nm}$ and a force resolution of $25 \mathrm{nN}$ under a $\times 50$ objective (see Methods). The MEMS device (Fig. 5a-d) is fabricated with an on-chip actuator and a loadcell spring in series. The sample is gripped between the loadcell and a pad rigidly connected to the substrate. When an actuator takes a step to the right, it pulls the loadcell and the specimen. Since the loadcell and the specimen are in series, the force on the sample can be obtained from the stiffness and the extension of the loadcell. Similarly, the specimen displacement can be obtained 
a
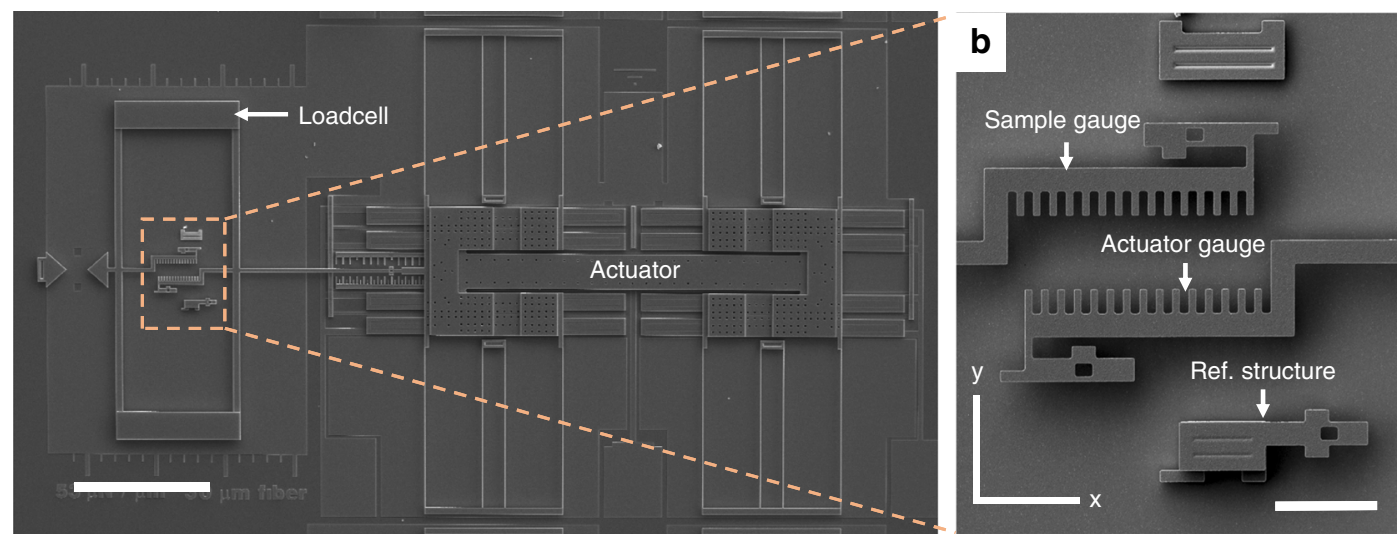

C
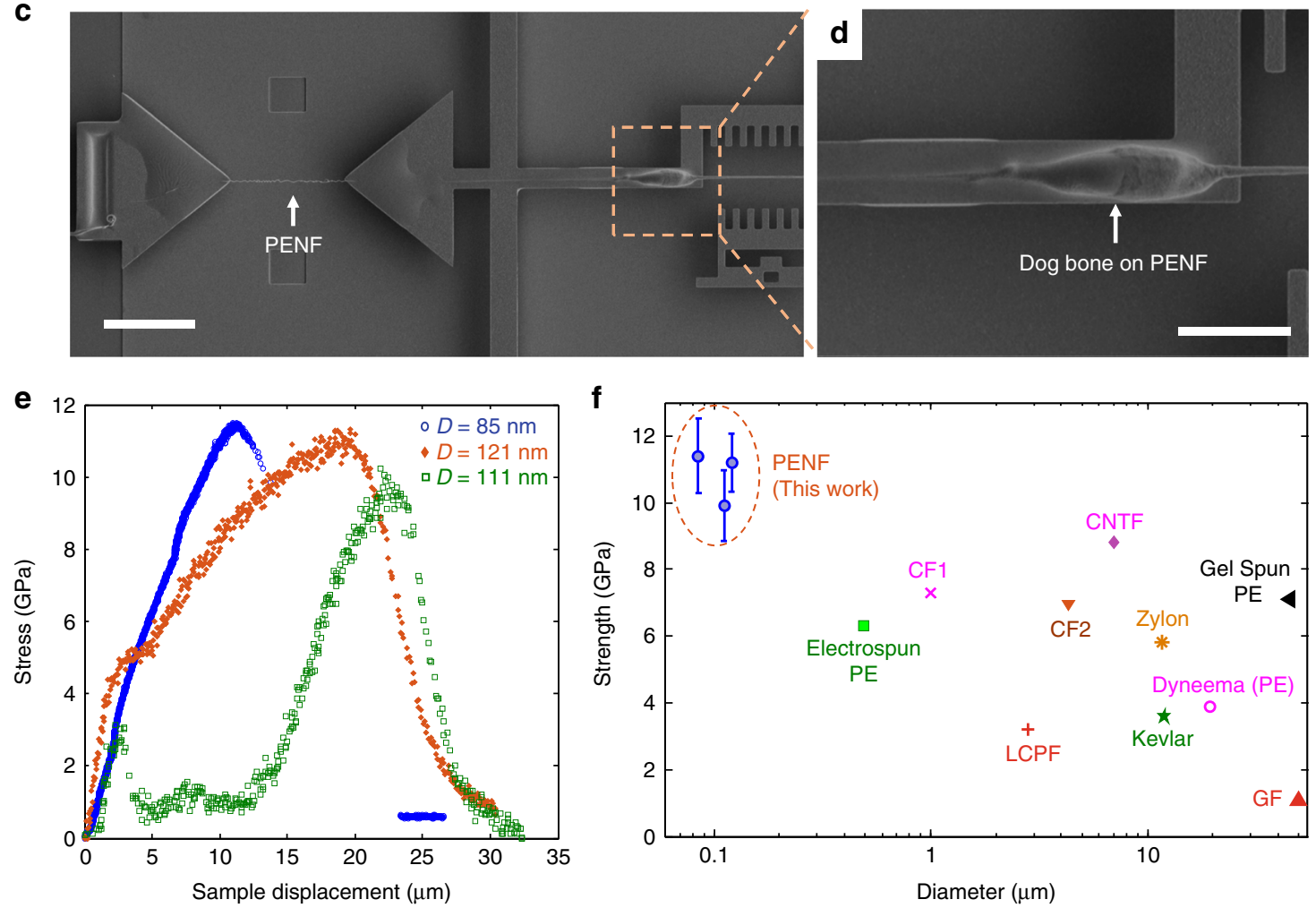

Fig. 5 Mechanical characterization of PENF. a-d SEM images of MEMS device used for tensile test of PENF. b Gauges for pattern matching to obtain sample displacement and loadcell extension. c A PENF gripped between two pads. $\mathbf{d}$ Dog bone shape on a PENF which provides additional grip due to mechanical locking. High contrast at the edges of dog bone is from charging of cured epoxy. e Stress-displacement curve. $\mathbf{f} \sigma_{\mathrm{ts}}$ of PENF compared to other fibers such as electrospun PE ${ }^{13}, \mathrm{CF}^{14}$ (Carbon fiber 1), CF2 (HexTow ${ }^{\circledR} /$ Hexcel), CNTF ${ }^{41}$ (Carbon nanotube fiber), PE ${ }^{42}$ (Gel-spun PE fiber), Zylon ${ }^{\circledast} /$ Toyobo, LCPF (Liquid crystal polymer fiber, Vectran ${ }^{\top M}$ / Kuraray), Kevlar ${ }^{\circledast}$ Du Pont, Dyneema ${ }^{\circledR}$ / Royal DSM, and GF ${ }^{16}$ (Graphene fiber). The error bars in PENF are calculated using uncertainty analysis (See Supplementary Note 5). Scale bars, $200 \mu \mathrm{m}$ (a), $25 \mu \mathrm{m}$ (b, c), and10 $\mu \mathrm{m}$ (d)

from the sample gauge displacement. After the mechanical measurement, the diameter and the length of the fiber were measured by SEM. A stress-displacement curve was generated, from which the $\sigma_{\text {ts }}$ was obtained.

In the tensile tests, it is challenging to grip the PENFs using adhesive because of the low surface energy of $\mathrm{PE}^{43}\left(31 \mathrm{~mJ} \mathrm{~m}^{-2}\right)$, and slip occur before they are tested to failure. We thus designed a dog bone shaped nanofiber (Fig. $5 \mathrm{c}, \mathrm{d}$ ) to provide additional mechanical locking (see Supplementary Note 6). However, even then, some slip still occurs, and thus the $E$ cannot be characterized accurately in our method. It should be noted that in Fig. 5e, the sample with $D=111 \mathrm{~nm}$ slips after the stress exceeds $3 \mathrm{GPa}$, then becoming taut until the dog bones provide the necessary grip.
The $\sigma_{\mathrm{ts}}$ of $11.4 \pm 1.1 \mathrm{GPa}$ was obtained for the nanofiber with diameter $85 \mathrm{~nm}$. To our knowledge, this is the highest measured $\sigma_{\text {ts }}$ for any polymer fiber reported including carbon fiber, Zylon ${ }^{\circledR}$, Kevlar $^{\circledR}$ and nylon fibers ${ }^{13-15,44}$ (Fig. 5f). Even though the theoretical $\mathrm{C}-\mathrm{C}$ bond strength (for chain scission) can be as high as $20 \mathrm{GPa}$, PE strength is limited by slip that initiates at chain ends $(6-8 \mathrm{GPa})^{45}$. The failure mode is a combination of chain slip and chain scission. A significantly minimized chain end density within the test length due to the local heating based fabrication technique and short gage length $(30 \mu \mathrm{m})$, comparable to the length of the PE molecule $(\sim 30-55 \mu \mathrm{m}$ for ultra-high molecular weight PE), explains the high strength. In addition, the diameter is not uniform over a length of $30 \mu \mathrm{m}$ (see Supplementary Fig. 22). For mechanically tested samples, the narrow region 
along the sample used for calculating the $\sigma_{\text {ts }}$ spans less than $1 \mu \mathrm{m}$ (see Supplementary Fig. 19). As this is much smaller than the length of a PE molecule, the likelihood of chain ends in the region is negligible. Therefore, these regions can sustain substantially larger stress than those limited by chain ends.

Two distinct failure mechanisms were observed when tested with low $\left(5.4 \mathrm{~N} \mathrm{~m}^{-1}\right)$ and high $\left(50.8 \mathrm{~N} \mathrm{~m}^{-1}\right)$ stiffness loadcells. The sample tested with the low stiffness loadcell exhibited ductile failure (Fig. 5e, $D=85 \mathrm{~nm}$ ), whereas that with the high stiffness loadcell experienced extreme necking, where the diameter was reduced to less than $10 \mathrm{~nm}$ (see Supplementary Fig. 20). This is because of the elastic strain energy $U=(1 / 2) k_{\mathrm{L}} \delta_{\mathrm{L}}^{2}=(1 / 2) F^{2} / k_{\mathrm{L}}$ stored in the loadcells. For a given load $F$, higher elastic strain energy is stored in the lower stiffness loadcell. For small $k_{\mathrm{L}}(5.4 \mathrm{vs}$. $\left.50.8 \mathrm{~N} \mathrm{~m}^{-1}\right), F$ drops slowly during displacements after the $\sigma_{\mathrm{ts}}$ is reached, increasing the likelihood of instability.

\section{Discussion}

In summary, we have demonstrated highly aligned and crystalline PENF with diameters of $10-100 \mathrm{~nm}$ fabricated by a localized heating and drawing method. Both SAED and micro Raman analyses clearly verify the high crystallinity and molecular alignment. Values of $k(T)$ from $20 \mathrm{~K}$ to $320 \mathrm{~K}$ are reported, where $k$ reaches up to $90 \mathrm{~W} \mathrm{~m}^{-1} \mathrm{~K}^{-1}$ at $130 \mathrm{~K}$. This suggests that PENF can be used as an efficient heat transfer material for cryogenic purposes particularly where an electrically insulating material is concurrently desired ${ }^{46}$. At low temperatures, $k$ increases as $\sim T^{1}$, which stems from the one dimensionality of phonon transport and extrinsic scattering (e.g., defects, boundary). Measurement of $\sigma_{\mathrm{ts}}$ using a MEMS device demonstrates that these nanofibers have ultra-high $\sigma_{\mathrm{ts}}$ up to $11.4 \pm 1.1 \mathrm{GPa}$. This is the highest $\sigma_{\mathrm{ts}}$ reported for any polymer fiber or composite.

The values we obtained for $\sigma_{\mathrm{ts}}$ and $k$ are critically dependent on the cross-section area, which is challenging to measure at this scale. As stated above and detailed in the Supplementary Note 7 (Supplementary Fig. 21), the cross-sections are non-circular. However, when calculating $\sigma_{\mathrm{ts}}$ and $k$ of PENF from the measured force and thermal conductance we assumed that the fibers have a circular cross-section, and used the larger dimension. Hence, the $k$ reported in Fig. $3 \mathrm{c}$ and e and $\sigma_{\text {ts }}$ reported in Fig. 5e and f should be taken as a lower bound for PENF.

The polymer nanofibers with lightweight-thermal-mechanical functionality could find general applications in aerospace and automotive systems, where a high strength-to-weight ratio (Supplementary Note 1) is desirable. With the multifunctionality, the polymer nanofibers are purely electrically insulating and biocompatible, which opens up unique applications in electronics and biology. These nanofibers provide highly effective heat removal in electronic systems, for example, heat sinks for electronic packages. Furthermore, integrating local heating as a secondary process on gel spun microfibers could potentially realize making larger scale PE nanofibers.

\footnotetext{
Methods

Localized heating/drawing to fabricate PENF. In this method, we first produce $\mathrm{PE} /$ decalin gel. Then, a sharp glass tip $(\sim 10 \mu \mathrm{m})$ is used to pull a PEMF at $90^{\circ} \mathrm{C}$. Tip drawing of PEMF from PE/decalin gel at an elevated temperature results in the unfolding of the chain lamellae into micro fibrils that are pulled taut between entanglements ${ }^{6}$. PEMF was then quenched to RT to reduce the relaxation of the aligned molecules. It was kept taut and attached to a sample collector, which is a bulk micromachined rectangular silicon frame with a square hole (see Supplementary Fig. 3). The pre-stressed PEMF on the sample collector was locally heated and stretched to nanoscale diameter using a home built microheater, which is a sharply bent tungsten micro wire with an etched tip. When a small segment of the PEMF was placed in proximity to the tip of the heater and heated close to the melting point, the pre-existing tensile stress immediately stretched the PEMF to a nanoscale diameter (see Supplementary Movie 1). A section of 80-100 $\mu \mathrm{m}$ long nanofiber was usually obtained in a PEMF using this two-step method. The
}

eventual diameter of the nanofiber can be controlled by tuning the pre-existing stress or the temperature of the heater.

Micro Raman characterization. We used a Reinshaw InVia Raman Microscope with a $532 \mathrm{~nm}$ laser. The sample was aligned using a low laser power of $2.5 \mathrm{~mW}$ to reduce the irradiation damage prior to characterization. An incident laser with 25 $\mathrm{mW}$ power was focused with a $\times 50$ objective onto a PE fiber suspended across a sample collector. A backscattered Raman spectrum was accumulated for $60 \mathrm{~s}$ at room temperature. The sample undergoes minimal structural damage during the characterization, which is verified by overlapping three separate Raman spectra collected at the same spot of a PENF sample (see Supplementary Fig. 10). The Raman spectra were collected with a spectral resolution of $0.9 \mathrm{~cm}^{-1}$ between 1000 $\mathrm{cm}^{-1}$ and $1500 \mathrm{~cm}^{-1}$.

Low dose cryo-TEM. Structural characterization of crystalline polymer is challenging under an electron beam as the radiation damages the sample crystallinity, which leads to fading and evolution of the diffraction spots into a ring pattern. The damage can be observed on a PE crystal at a dose as low as $25 \times 10^{-4} \mathrm{C} \mathrm{cm}^{-2}$, and complete loss of Bragg spots occurs at a critical dose of $0.01 \mathrm{C} \mathrm{cm}^{-2}$ at an accelerating voltage of $100 \mathrm{kV}^{30}$. The critical dose can be increased by reducing the temperature; in PE crystals it can be increased by a factor of 2.5 by cooling the sample from RT down to $100 \mathrm{~K}^{30}$. To reduce the electron beam damage and characterize morphological and structural features, we used low dose cryo-TEM. The sample was cooled to $100 \mathrm{~K}$ using a liquid nitrogen cooled sample holder. The sample was inspected at a low dose of $8 \times 10^{-5} \mathrm{C} \mathrm{cm}^{-2}$, whereas selected aperture electron diffraction (SAED) patterns were obtained at a dose close to the critical dose. Bright field images and SAED patterns were obtained at $80 \mathrm{kV}$.

Phonon dispersion calculation. To obtain the phonon dispersion of aligned PE chain bundles, we performed MD simulations to generate the trajectory. The condensed-phase optimized molecular potentials for atomistic simulation studies (COMPASS $^{47}$ all-atom potential is used to model the PE and a timestep of $0.5 \mathrm{fs}$ is employed. In the simulation domain, there are 4 chains in the unit cell and 400 $\mathrm{CH}_{2}$ segments in each chain with periodic boundary condition applied in all three spatial directions. After structural minimization, the simulation first runs at $2 \mathrm{~K}$ in the NVT (constant volume and temperature) ensemble, and then runs in the NVE (constant volume and energy) ensemble for 50 ps to produce the trajectory. The low temperature is chosen to reduce the noise and increase the contrast of the dispersion relation. During the NVE run, the velocity of every backbone carbon atom is recorded every $5 \mathrm{fs}$ in a two-dimensional matrix. Then, a two-dimensional Fourier transform of the atomic velocity of one carbon atom in the unit cell is performed:

$$
\Phi\left(\omega, \mathbf{k}_{\mathrm{v}}\right)=\sqrt{\sum_{\alpha}^{3}\left|\frac{1}{N} \sum_{n=0}^{N-1} e^{i \frac{\eta_{N}}{\mathbf{k}_{\mathrm{v}}}} \int v_{\alpha}(n, t) e^{-\mathrm{i} \omega t} \mathrm{dt}\right|^{2}}, \alpha=x, y, z
$$

where $v_{\alpha}(n, t)$ is the atomic velocity, $\omega$ is the the frequency, $\mathbf{k}_{\mathrm{v}}$ is the wavevector, $n$ is the index of repeating unit along chain direction, and $N$ is the number of the repeating unit. Next, the phonon dispersion relation is calculated by averaging the two-dimensional Fourier transform results of all the backbone atoms in the unit cell. The obtained dispersion plot, which is essentially a two-dimensional contour, is digitized and fitted with a polynomial (see Fig. 4b). We have also used the same method to calculate the dispersion of a standalone single PE chain, and the obtained dispersion relation agrees well with other calculations in the literature ${ }^{48}$ At the low temperature limit, where anharmonic phonon scattering is weak, we assume that extrinsic scattering will cause the phonon mean free path be the same for all phonon modes.

Stress and displacement measurements. After the sample was mounted and aligned onto the MEMS device, measurements were carried out on a custom designed probe station equipped with a long working distance microscope on a vibration isolation table at RT. The actuator was actuated at 1 step per second while load cell displacements were optically imaged with a $\times 50$ objective $(\mathrm{NA}=0.55)$ using a charge coupled device (CCD) camera at 1 frame per second and stored for data analysis. Images were analyzed to measure load cell displacements with resolution of $4 \mathrm{~nm}$ using sub pixel pattern matching. Load cell displacement measurements were used to calculate force and sample displacement. The force exerted on the sample is $F=k_{\mathrm{L}} \times\left(x_{\text {actuator gauge }}-x_{\text {sample gauge }}\right)$. After the diameter $(D)$ was measured using SEM, stress was calculated as $\sigma=4 F /\left(\pi D^{2}\right)$. Sample displacement was obtained as $u=\left(x_{\text {sample gauge }}-x_{\text {ref structure }}\right)$.

Sub pixel pattern matching for nanoscale resolution using optical microscopy Sub pixel pattern matching is used to obtain nanoscale resolution of displacement from optical images ${ }^{49,50}$. Direct measurement is diffraction limited, however, this can be circumvented by comparing two images and obtain displacement resolution smaller than a pixel. In sub-pixel pattern matching, a predetermined 2D pattern of a first image is iteratively searched in an expected window in subsequent images. Pattern positions $(x, y$, and $\theta)$ with the highest match score are recorded. Sub pixel 
displacement resolution is obtained by interpolating a pixel (bilinear here) on discrete structures such as edges or lines where a sharp contrast is achieved. Each pixel has a greyscale value from $2^{n}$ possible values where $n$ is the number of bits per pixel ( $n=8$ in our case). A perfect edge with white (1) on one side and black ( 0$)$ on other in a pixel could be interpolated with $2^{n}$ intensity values. In an ideal scenario with no noise and perfect contrast, a resolution of pixel $/ 2^{n}$ can be achieved. This would give $0.5 \mathrm{~nm}$ displacement resolution for an objective of $\times 50$ (pixel length is $121 \mathrm{~nm}$ ). Considering noise, we achieved pixel/30 i.e., $\sim 4 \mathrm{~nm}$ resolution (1 standard deviation) as aided by a large number of well-defined edges as provided here by the comb structures.

Data availability. The data that support the findings of this study are available in Supplementary Information and from the corresponding authors upon reasonable request.

Received: 19 September 2017 Accepted: 27 March 2018

Published online: 25 April 2018

\section{References}

1. Mark, J. E. Physical properties of polymers handbook. (Springer, New York, 2007).

2. Cunniff, P. M. et al. Mechanical and thermal properties of dragline silk from the spider Nephila clavipes. Polym. Adv. Technol. 5, 401-410 (1994).

3. Choy, C. L., Wong, Y. W., Yang, G. W. \& Kanamoto, T. Elastic modulus and thermal conductivity of ultradrawn polyethylene. J. Polym. Sci. Part B 37, 3359-3367 (1999).

4. Wang, X., Ho, V., Segalman, R. A. \& Cahill, D. G. Thermal conductivity of high-modulus polymer fibers. Macromolecules 46, 4937-4943 (2013).

5. Smith, P. \& Lemstra, P. J. Ultra-high-strength polyethylene filaments by solution spinning/drawing. J. Mater. Sci. 15, 505-514 (1980).

6. Shen, S., Henry, A., Tong, J., Zheng, R. \& Chen, G. Polyethylene nanofibres with very high thermal conductivities. Nat. Nanotechnol. 5, 251-255 (2010)

7. Li, P., Hu, L., McGaughey, A. J. H. \& Shen, S. Crystalline polyethylene nanofibers with the theoretical limit of Young's modulus. Adv. Mater. 26 1065-1070 (2014).

8. Zhang, T., Wu, X. \& Luo, T. Polymer nanofibers with outstanding thermal conductivity and thermal stability: fundamental linkage between molecular characteristics and macroscopic thermal properties. J. Phys. Chem. C. 118, 21148-21159 (2014).

9. Edie, D. D. The effect of processing on the structure and properties of carbon fibers. Carbon N. Y 36, 345-362 (1998).

10. Johnson, D. J. Structure-property relationships in carbon fibres. J. Phys. D 20, 286-291 (1987).

11. MInus, M. \& Kumar, S. The processing, properties, and structure of carbon fibers. JOM 57, 52-58 (2005).

12. Ma, J. et al. Thermal conductivity of electrospun polyethylene nanofibers. Nanoscale 7, 16899-16908 (2015).

13. Park, J. H. \& Rutledge, G. C. Ultrafine high performance polyethylene fibers. J. Mater. Sci. 53, 3049-3063 (2017).

14. Şahin, K. et al. High strength micron size carbon fibers from polyacrylonitrile-carbon nanotube precursors. Carbon 77, 442-453 (2014).

15. Yao, J., Bastiaansen, C. \& Peijs, T. High strength and high modulus electrospun nanofibers. Fibers 2, 158-186 (2014).

16. Xin, G. et al. Highly thermally conductive and mechanically strong graphene fibers. Science 349, 1083-1087 (2015).

17. Perkins, T. T. et al. Single polymer dynamics in an elongational flow. Science 276, 2016-2021 (1997)

18. Strobl, G. R. \& Hagedorn, W. Raman spectroscopic method for determining the crystallinity of polyethylene. J. Polym. Sci. Part B 16, 1181-1193 (1978).

19. W. Bunn, B. C. The crystal structure of long-chain normal paraffin hydrocarbons. The 'shape' of the $\mathrm{CH} 2$ group. Trans. Faraday Soc. 35, 482-491 (1939).

20. Kawaguchi, A., Matsui, R. \& Kobayashi, K. The crystal structure of polyethylene at $4.5^{\circ}$ K. Bull. Inst. Chem. Res. Kyoto Univ. 55, 217-226 (1977).

21. Zhang, T. \& Luo, T. High-contrast, reversible thermal conductivity regulation utilizing the phase transition of polyethylene nanofibers. ACS Nano 7, 7592-7600 (2013).

22. Frank, F. C., Keller, A., O 'Connor, A. \& H. Wills, H. Deformation processes in polyethylene interpreted in terms of crystal plasticity. Phil. Mag. 3, 64-74 (1958).

23. Kiho, H., Peterlin, A. \& Geil, P. H. Polymer deformation. VI. Twinning and phase transformation of polyethylene single crystals as a function of stretching direction. J. Appl. Phys. 35, 1599-1605 (1964).

24. Preedy, J. E. \& Wheeler, E. J. A study of twinning in polyethylene. J. Mater. Sci. 12, 810-815 (1977).
25. Li, D. et al. Thermal conductivity of individual silicon nanowires. Appl. Phys. Lett. 83, 2934-2936 (2003).

26. Hippalgaonkar, K. et al. Fabrication of microdevices with integrated nanowires for investigating low-dimensional phonon transport. Nano Lett. 10 4341-4348 (2010)

27. Hochbaum, A. I. et al. Enhanced thermoelectric performance of rough silicon nanowires. Nature 451, 163-167 (2008).

28. Chen, R. et al. Thermal conductance of thin silicon nanowires. Phys. Rev. Lett. 101, 105501 (2008)

29. Lee, S. et al. Anomalously low electronic thermal conductivity in metallic vanadium dioxide. Science 355, (2017).

30. Grubb, D. T. \& Groves, G. W. Rate of damage of polymer crystals in the electron microscope: Dependence on temperature and beam voltage. Philos. Mag. 24, 815-828 (1971).

31. Yang, J. et al. Contact thermal resistance between individual multiwall carbon nanotubes. Appl. Phys. Lett. 96, 23109-224308 (2010).

32. $\mathrm{Xu}, \mathrm{Y}$. et al. Nanostructured polymer films with metal-like thermal conductivity. Preprint at arXiv:1708.06416 (2017).

33. Ronca, S., Igarashi, T., Forte, G. \& Rastogi, S. Metallic-like thermal conductivity in a lightweight insulator: solid-state processed ultra high molecular weight polyethylene tapes and films. Polymer 123, 203-210 (2017).

34. Zhu, B. et al. Novel polyethylene fibers of very high thermal conductivity enabled by amorphous restructuring. ACS Omega 2, 3931-3944 (2017).

35. Gofryk, K. et al. Anisotropic thermal conductivity in uranium dioxide. Nat. Commun. 5, 4551 (2014).

36. Broido, D. A., Malorny, M., Birner, G., Mingo, N. \& Stewart, D. A. Intrinsic lattice thermal conductivity of semiconductors from first principles. Appl. Phys. Lett. 91, 231922 (2007).

37. Zhang, T. \& Luo, T. Morphology-influenced thermal conductivity of polyethylene single chains and crystalline fibers. J. Appl. Phys. 112, 94304 (2012).

38. Kittel, C. Introduction to solid state physics. (Wiley, 2005).

39. deBoer, M. P. et al. High-performance surface-micromachined inchworm actuator. J. Micro. Syst. 13, 63-74 (2004).

40. Naraghi, M., Ozkan, T., Chasiotis, I., Hazra, S. S. \& de Boer, M. P. MEMS platform for on-chip nanomechanical experiments with strong and highly ductile nanofibers. J. Micromech. Microeng. 20, 125022 (2010).

41. Koziol, K. et al. High-performance carbon nanotube fiber. Science 318, 1892-1895 (2007)

42. Van der Werff, H. \& Pennings, A. J. Tensile deformation of high strength and high modulus polyethylene fibers. Colloid Polym. Sci. 269, 747-763 (1991)

43. Brewis, D. M. \& Briggs, D. Adhesion to polyethylene and polypropylene. Polymer 22, 7-16 (1981).

44. Zussman, E., Burman, M., Yarin, A. L., Khalfin, R. \& Cohen, Y. Tensile deformation of electrospun nylon-6,6 nanofibers. J. Polym. Sci. Part B 44, 1482-1489 (2006).

45. O'Connor, T. C. \& Robbins, M. O. Chain ends and the ultimate strength of polyethylene fibers. ACS Macro Lett. 5, 263-267 (2016)

46. Yamanaka, A., Takao, T., Yamanaka, A. \& Takao, T. Thermal conductivity of high-strength polyethylene fiber and applications for cryogenic use. ISRN Mater. Sci. 2011, 1-10 (2011).

47. Sun, H. COMPASS: An ab initio force-field optimized for condensed-phase applications overview with details on alkane and benzene compounds. J. Phys Chem. B 102, 7338-7364 (1998).

48. Henry, A. \& Chen, G. High thermal conductivity of single polyethylene chain using molecular dynamics simulations. Phys. Rev. Lett. 101, 235502 (2008).

49. Shroff, S. S., Ansari, N., Robert Ashurst, W. \& de Boer, M. P. Rate-state friction in microelectromechanical systems interfaces: experiment and theory. J. Appl. Phys. 116, 244902 (2014).

50. Corwin, A. D. \& de Boer, M. P. Frictional aging and sliding bifurcation in monolayer-coated micromachines. J. Micro. Syst. 18, 250-262 (2009).

\section{Acknowledgements}

This work is supported by the US National Science Foundation (NSF) under award CMMI-1334630, the US Air Force Office of Scientific Research, Young Investigator Program under award FA9550-17-1-0141 (for thermoreflectance imaging), the Dupont Young Professor Award and the American Chemical Society under award PRF\# 54129 DNI10 (for numerical simulations) and A*STAR-SERC Pharos program under award 1527200018 (for thermal device fabrication and analysis). We thank Dr. Mohammad Naraghi (Texas A\&M University) for helpful discussions.

\section{Author contributions}

S.S., M.dB., and R.S. conceived and designed the experiments. R.S. performed sample fabrication, structural characterization, and thermal and mechanical characterizations. P.L. contributed to sample fabrication and thermal characterization. B.C., R.S. and S.C performed thermoreflectance imaging. T.Z., X.W., Z.L., and T.L. performed numerical 
simulations. K.H. fabricated micro thermal devices. R.S., M.dB., and S.S. wrote the paper. All authors discussed the results and commented on the manuscript. S.S. and M.dB. supervised the research.

\section{Additional information}

Supplementary Information accompanies this paper at https://doi.org/10.1038/s41467018-03978-3.

Competing interests: The authors declare no competing interests.

Reprints and permission information is available online at http://npg.nature.com/ reprintsandpermissions/

Publisher's note: Springer Nature remains neutral with regard to jurisdictional claims in published maps and institutional affiliations. (c) (i) Open Access This article is licensed under a Creative Commons Attribution 4.0 International License, which permits use, sharing, adaptation, distribution and reproduction in any medium or format, as long as you give appropriate credit to the original author(s) and the source, provide a link to the Creative Commons license, and indicate if changes were made. The images or other third party material in this article are included in the article's Creative Commons license, unless indicated otherwise in a credit line to the material. If material is not included in the article's Creative Commons license and your intended use is not permitted by statutory regulation or exceeds the permitted use, you will need to obtain permission directly from the copyright holder. To view a copy of this license, visit http://creativecommons.org/ licenses/by/4.0/.

(C) The Author(s) 2018 